\title{
Radiology and nuclear medicine: advancing together in the era of precision medicine
}

\author{
Arturo Chiti ${ }^{1,2} \cdot$ Yves Menu $^{3}$ (D) \\ Published online: 15 January 2020 \\ (C) Springer-Verlag GmbH Germany, part of Springer Nature, and European Society of Radiology 2019
}

Nuclear medicine and radiology are recognised as separate medical specialties in many European countries. The two have distinct differences, but also similarities as they both offer diagnostic procedures and therapeutic techniques. Moreover, both specialties need to educate professionals working in the respective disciplines.

Medicine is nowadays evolving at an incredible pace and these two disciplines are at the forefront of several fields, above all artificial intelligence (AI). AI applied to imaging is a clear example of how close imaging specialties, radiology, and nuclear medicine are to each other. Neural networks will be able to recognise images within a particular clinical context, integrating with epidemiology and genomics data.

The way images are generated will be important for their interpretation, but not so much to the extent that this would then justify the generation of different kinds of neural networks.

The combination of two different imaging modalities (hybrid imaging) is already a field in which the specialist's knowledge of both disciplines is beneficial for the patient. Several centres are performing positron emission tomography (PET) with contrastenhanced computed tomography (CT) and PET with diagnostic magnetic resonance (MR) on a routine basis. In all these cases,

This Editorial is published simultaneously in European Journal of Nuclear Medicine and Molecular Imaging; see https://doi.org/10.1007/ s00259-019-04565-4.

Arturo Chiti

arturo.chiti@hunimed.eu

Yves Menu

yves.menu@aphp.fr

1 Department of Biomedical Sciences, Humanitas University, Milan, Italy

2 Unit of Nuclear Medicine, Humanitas Research Hospital, Milan, Italy

3 Department of Diagnostic and Interventional Radiology, Saint Antoine Hospital, APHP-Sorbonne University, Paris, France professionals take care of patients by collaborating with colleagues from 'the other field', or are already certified in both disciplines. Communication of knowledge and experience is the key for successful collaboration and represents a benefit not only for patients, but also for the health care providers involved in imaging.

Participation in multidisciplinary meetings is another example in which imaging specialists are called together to discuss the best diagnostic and therapeutic path for each patient. Moreover, both radiology and nuclear medicine are capable of treating patients with several diseases using interventional procedures and radiopharmaceutical therapy. Intra-arterial treatments with radionuclides are examples of how 'hybrid' can be successful in a therapy, not only in imaging.

Whether we are dealing with functional or morphological imaging, interventional procedures or radiopharmaceutical therapy, similarities between the two specialties are far more important than the differences. The common language allows for a strong interaction and facilitates the transfer of knowledge and experience within the larger field of medicine. This situation has the potential to benefit professionals from both specialties.

Our scientific results, guidelines and educational papers are published in two well-respected journals, the European Journal of Nuclear Medicine and European Radiology, along with journals in their respective 'families'. Papers dealing with functional imaging and hybrid imaging are published in both journals, after the fruitful collaboration of professionals from both radiology and nuclear medicine.

Science is the result of interactions between several professionals with different educations and experiences. As Editors-inChief of the journals representing the scientific associations of European Radiology and European Journal of Nuclear Medicine and Molecular Imaging, we are convinced that collaboration must be tightened at the scientific level. Therefore, we decided to join efforts for the common goal of enriching the respective disciplines. 
The first step in this process is to invite experts from both sides to join the Editorial Boards to act as ambassadors and serve as reviewers in areas of interdisciplinary research.

We hope that all professionals in the fields of radiology and nuclear medicine will join our efforts to keep raising the scientific level — not only of our journals but of the whole community.

Funding information The authors state that this work has not received any funding.

\section{Compliance with ethical standards}

Guarantor Not applicable

Conflict of interest The authors of this manuscript declare no relationships with any companies, whose products or services may be related to the subject matter of the article.
Statistics and biometry No complex statistical methods were necessary for this paper.

Informed consent Not applicable

Ethical approval Institutional Review Board approval was not required because the paper is an Editorial.

Study subjects or cohorts overlap: Not applicable

\section{Methodology}

- Not applicable

Publisher's note Springer Nature remains neutral with regard to jurisdictional claims in published maps and institutional affiliations. 